\title{
123I-Iodobenzovesamicol SPECT Imaging of Cholinergic Systems in Dementia with Lewy Bodies
}

\author{
Joachim Mazère ${ }^{1-3}$, Frédéric Lamare ${ }^{1-3}$, Michele Allard ${ }^{1-4}$, Philippe Fernandez ${ }^{1-3}$, and Willy Mayo ${ }^{1,2}$ \\ ${ }^{1}$ Université de Bordeaux, INCIA, UMR 5287, Bordeaux, France; ${ }^{2}$ CNRS, INCIA, UMR 5287, Bordeaux, France; ${ }^{3}$ Service de Médecine \\ Nucléaire, CHU de Bordeaux, Bordeaux, France; and ${ }^{4}$ EPHE, Paris, France
}

Cholinergic alterations in dementia with Lewy bodies (DLB) have been widely documented in postmortem studies, whereas in vivo studies are sparse, particularly at the subcortical level. We used 123/-iodobenzovesamicol, a SPECT radiotracer of the vesicular acetylcholine transporter, to evaluate in vivo in DLB the integrity of the 3 main cholinergic pathways - the Ch1 (septohippocampal), the Ch4 (innominatocortical), and the Ch5 (pontothalamic) cholinergic pathwaysas well as the striatal cholinergic interneurons. In addition, we assessed the involvement of the cholinergic system in cognitive and neuropsychiatric disorders in DLB patients. Methods: Twelve healthy volunteers (median age, $72 \mathrm{y}$; interquartile range, $6.25 \mathrm{y}$ ) and 11 DLB patients (median age, $76 \mathrm{y}$; interquartile range, $10.50 \mathrm{y}$ ) underwent a dynamic ${ }^{123}$-iodobenzovesamicol SPECT scan and an MRI scan. MR images were automatically segmented, providing the volumes of several regions of interest, including the striatum and cholinergic terminals in Ch1 (hippocampus), Ch4 (cortical lobes), and Ch5 (thalamus). For each region of interest and each subject, pharmacokinetic modeling allowed calculation of the nondisplaceable binding potential $\left(\mathrm{BP}_{\mathrm{ND}}\right)$ values for the binding of ${ }^{123}$-iodobenzovesamicol to the vesicular acetylcholine transporter. A neuropsychological evaluation of participants was performed with the Mini-Mental State Examination and the Grober-Buschke, Set, visual discrimination, Benton, and Wechsler tests, and cognitive fluctuations and apathy were also assessed. Results: Compared with $\mathrm{BP}_{\mathrm{ND}}$ values for healthy subjects, $\mathrm{BP}_{\mathrm{ND}}$ values for DLB patients were significantly lower in the Ch4 terminal regions of the anterior cingulate cortex and the superior and inferior parietal cortices $(P=0.0006,0.0015$, and 0.0023 , respectively), in the Ch5 terminal region of the thalamus $(P=0.0003)$, and in the striatum $(P=0.0042)$. All of the neuropsychological test scores were significantly lower in DLB patients than in healthy subjects. Four DLB patients with apathy and 4 DLB patients without apathy were identified. For the anterior cingulate cortex, compared with $\mathrm{BP}_{\mathrm{ND}}$ values in healthy subjects, $\mathrm{BP}_{\mathrm{ND}}$ values were significantly lower in patients with apathy $(P=0.004)$ and were unchanged in patients without apathy. Conclusion: Our results confirm the existence in DLB of cholinergic alterations, reaching both cortical and subcortical levels, including the Ch5 pathway and the striatum. Alterations in cholinergic transmission in the anterior cingulate cortex could be closely associated with the development of apathy.

\footnotetext{
Received Mar. 23, 2016; revision accepted Jun. 29, 2016.

For correspondence or reprints contact: Joachim Mazère, Université de Bordeaux, INCIA, UMR 5287, 146 Rue Léo Saignat, 33076 Bordeaux Cedex, France.

E-mail: joachim.mazere@chu-bordeaux.fr

Published online Jul. 28, 2016.

COPYRIGHT (C 2017 by the Society of Nuclear Medicine and Molecular Imaging.
}

Key Words: ${ }^{123}$-iodobenzovesamicol; SPECT; cholinergic pathways; dementia with Lewy Bodies; apathy

J Nucl Med 2017; 58:123-128

DOI: $10.2967 /$ jnumed.116.176180

D ementia with Lewy bodies (DLB), which is claimed to be the second most common type of neurodegenerative dementia after Alzheimer disease (AD), represents $15 \%$ of degenerative dementias (1). A central feature of DLB is a progressive cognitive decline associated with several criteria, including the core features fluctuating cognition, visual hallucinations, and spontaneous parkinsonism as well as suggestive features, such as sleep disturbances (2). Only 2 core features or 1 core feature and 1 suggestive feature are sufficient to diagnose probable DLB, resulting in large variability in the clinical expression of DLB. Thus, clinical subtypes of DLB may reflect heterogeneity in neurochemical alterations. Indeed, besides dopaminergic alterations (3), cholinergic systems were shown to be impaired in DLB, and the benefit of cholinesterase inhibitor drugs for neuropsychiatric and cognitive symptoms confirmed the cholinergic hypothesis for DLB (2).

The status of the presynaptic cholinergic system in DLB has been well documented in postmortem studies, which have demonstrated a profound and widespread reduction in choline acetyltransferase activity $(4,5)$. However, in vivo studies of DLB are sparse; a decrease in acetylcholine esterase (AChE) activity in cortical regions has been shown (6-8), but the integrity of subcortical cholinergic systems remains poorly documented. Indeed, to date, only one PET study explored the cholinergic innervation of the thalamus in DLB patients (9); the integrity of striatal cholinergic interneurons has never been assessed, probably because the AChE radiotracers classically used in PET studies have been reported to be ineffective for assessing striatal cholinergic activity (9). ${ }^{123}$ I-iodobenzovesamicol is a SPECT radiotracer that binds selectively to a presynaptic cholinergic marker, the vesicular acetylcholine transporter (VAChT), thereby serving as an in vivo marker of presynaptic cholinergic terminal density. ${ }^{123}$ I-iodobenzovesamicol proved to be a successful radiotracer in the evaluation of cortical as well as subcortical cholinergic status in vivo for various neurologic disorders (10-13).

To determine whether there are differences in cholinergic alterations at the cortical and subcortical levels in vivo in DLB, we evaluated the integrity of the 3 main cholinergic pathways- the Ch1 (septohippocampal), the $\mathrm{Ch} 4$ (innominatocortical), and the $\mathrm{Ch} 5$ (pontothalamic) pathways - as well as the striatal cholinergic interneurons in DLB patients and age-matched healthy control subjects. 
In addition, using a patient subgroup-based study, we evaluated the involvement of the cholinergic system in cognitive and neuropsychiatric disorders in DLB patients.

\section{MATERIALS AND METHODS}

\section{Subjects}

The participants were 12 healthy volunteers (median age, $72 \mathrm{y}$; interquartile range, $6.25 \mathrm{y}$ ) without neuropsychiatric disorders, as determined in a screening interview by an experienced senior neuropsychologist, and 11 patients (median age, $76 \mathrm{y}$; interquartile range, $10.50 \mathrm{y}$ ), recruited according to revised international consensus criteria for the clinical diagnosis of DLB (2). A diagnosis of probable DLB was based on at least 1 core feature plus 1 suggestive feature or 2 core features, and a diagnosis of possible DLB was based on 1 core feature or 1 suggestive feature. For grading of the cognitive state of the participants, all were evaluated with the following neuropsychological tests: the Mini-Mental State Examination (14), the Grober-Buschke test (15), the Benton test (16), the visual discrimination test, the Set test described by Isaacs et al. (17), and the Wechsler Adult Intelligence Scale (18). Cognitive fluctuations were assessed with the examination described by Walker et al. (19), and apathy was assessed with the Neuropsychiatric Inventory (NPI) (20). For technical reasons, 3 patients were not able to undergo the cognitive fluctuation and apathy examinations, and 1 patient could not undergo the Wechsler examination.

The study was initiated after approval of the protocol by the Institutional Human Ethics Committee (registration number 2009/08) and radioactive drug approval by the National Health Product Safety Agency. After being presented with the basic design of the study and informed that they could withdraw from the investigation at any time, all participants provided written informed consent.

\section{Radiochemistry}

${ }^{123}$ I-iodobenzovesamicol was prepared by oxidative radioiodination of the respective (-)-5-tributyltin precursor, as the (-)-5-position isomer of iodobenzovesamicol (levogyre form) exhibits the greatest affinity and selectivity for the VAChT (21). Synthesis and quality control were achieved as previously described (12). Briefly, after radioiodination, ${ }^{123}$ I-iodobenzovesamicol was purified by high-pressure liquid chromatography and Sep-Pack $\mathrm{C}_{18}$ classic cartridges (Waters) and filtered, leading to a $10-\mathrm{mL}$ sterile solution of ${ }^{123}$ I-iodobenzovesamicol. Before injection, the solution was determined to be pyrogen-free with an Endosafe-PTS portable test system (Charles River Laboratories).

\section{Scanning Protocol}

Anatomic MRI. For anatomic colocalization with the SPECT data, participants underwent a 3-dimensional T1-weighted MRI sequence (repetition time, $7.1 \mathrm{~ms}$; echo time, $3.5 \mathrm{~ms} ; 8^{\circ}$ flip angle; field of view, $256 \times$ $256 \mathrm{~mm}$ [to cover the whole brain, yielding 228 slices; 1-mm slice thickness; $1-\mathrm{mm}^{3}$ isotropic voxel size]) with a 1.5-T MRI system (Philips).

SPECT/CT Imaging. SPECT imaging was performed on a SPECT/CT SYMBIA T2 camera (Siemens) equipped with low-energy thin-section collimators. Participants were given $400 \mathrm{mg}$ of potassium perchlorate orally $30 \mathrm{~min}$ before and $24 \mathrm{~h}$ after imaging. After an intravenous injection of $227.8 \pm 36.8$ (mean $\pm \mathrm{SD}$ ) MBq of ${ }^{123}$ I-iodobenzovesamicol, dynamic SPECT images were acquired over $8 \mathrm{~h}$ at 6 different times: 5 frames of 6 min starting at the time of injection and 2 frames of $15 \mathrm{~min}$ at 2, 3, 5, and $8 \mathrm{~h}$ after injection ( 64 projections with a matrix of $64 \times 64$ over $360^{\circ}$ ). Between each acquisition, the participants were allowed to rest outside the gantry.

SPECT images were reconstructed on a $64 \times 64$ matrix with a Flash (Siemens) 3-dimensional iterative reconstruction (4 iterations, 8 subsets) and a voxel size of $6.8 \times 6.8 \times 6.8 \mathrm{~mm}$. Attenuation correction was based on the CT image, and decay correction and normalization for frame duration were also performed. All of the frames were rigidly registered together and with the CT image to ensure that the participant's brain was in the exact same position on all of the images during the creation of the dynamic SPECT dataset.

\section{SPECT Data Processing}

SPECT Data Registration to MR Images. Each participant's CT and MR images were rigidly coregistered, and the transformation parameters were subsequently applied to the SPECT dataset, providing a 4dimensional dynamic SPECT image registered to the MR image.

Partial-Volume Effect Correction of Dynamic SPECT Dataset. The MR image-coregistered SPECT image was corrected for the partialvolume effect with the MRI-based method of Müller-Gärtner et al. (22), implemented in PMOD software (version 3.3; PMOD Technologies). For each participant, the MR image was automatically segmented into gray matter, white matter, and cerebrospinal fluid probability maps, and then the partial-volume effect correction procedure corrected the SPECT data for gray matter spill-out and white matter spill-in.

\section{Time-Activity Curve Calculations}

For each participant, the MR image-registered and partial-volume effect-corrected dynamic SPECT data were used for the derivation of regional time-activity curves. The MR image was automatically segmented with Freesurfer software (23), providing the volumes and labels of 12 gray matter regions of interest (ROIs), including the striatum, thalamus, and various cortical structures (prefrontal cortex, motor and premotor cortices, occipital cortex, superior parietal cortex, inferior parietal cortex, temporal cortex, anterior cingulate cortex (ACC), posterior cingulate cortex, hippocampus, and cerebellar hemispheres). The PMOD software was used to subsequently apply this gray matter template to each frame of the registered and partial-volume effect-corrected dynamic SPECT data to obtain average decay-corrected regional activities, which were plotted against time to derive regional time-activity curves.

\section{Quantification of ${ }^{123}$ |-lodobenzovesamicol SPECT Data}

The reference method for the noninvasive quantification of ${ }^{123} \mathrm{I}$ iodobenzovesamicol data was used (24), that is, a complete multilinear reference tissue model pharmacokinetic analysis (25), with the cerebellar hemispheres as reference regions (26). This method allowed us to calculate the nondisplaceable binding potential $\left(\mathrm{BP}_{\mathrm{ND}}\right)$ values for the binding of ${ }^{123}$ I-iodobenzovesamicol to the vesicular acetylcholine transporter for each participant and each $\mathrm{ROI}$. The value of the $\mathrm{BP}_{\mathrm{ND}}$ parameter is proportional to the density of receptor sites, in this case, VAChT binding sites.

\section{Statistical Analysis}

Mann-Whitney $U$ tests were used to test differences in $\mathrm{BP}_{\mathrm{ND}}$ values in each ROI between healthy subjects and DLB patients. A KruskalWallis 1-way ANOVA followed by a Mann-Whitney $U$ test was used to assess differences between healthy subjects and DLB patient subgroups. The results of the present study included corrections for multiple comparisons; 11 regions were examined, and a conservative multiple comparison with the Bonferroni adjustment indicated that the significance level should be set at a $P$ value of less than 0.0045 . Statistical analyses were performed with Statistica software (version 9; Statsoft).

\section{RESULTS}

\section{Subject Characteristics}

The ratio of men to women was higher for DLB patients (7:4) (Table 1) than for control subjects (3:9). Of the 8 DLB patients evaluated with the NPI, a subgroup of 4 patients with apathy (NPI score different from 0 ) and a subgroup of 4 patients without apathy (NPI score of 0 ) were identified. Eight of the 8 patients evaluated with the examination described by Walker et al. (19) exhibited a score different from 0 , so that the identification of patient subgroups with and without cognitive fluctuations was not possible. 
TABLE 1

Patient Demographics and Cognitive Fluctuation and Apathy Scores*

\begin{tabular}{|c|c|c|c|c|c|}
\hline Patient & Condition & Age (y) & Sex & Cognitive fluctuation score/22 & Apathy score \\
\hline 1 & Possible DLB & 68 & $\mathrm{~F}$ & NA & NA \\
\hline 2 & Possible DLB & 81 & M & NA & NA \\
\hline 3 & Probable DLB & 78 & $\mathrm{~F}$ & NA & NA \\
\hline 4 & Probable DLB & 70 & M & 7 & 8 \\
\hline 5 & Probable DLB & 76 & M & 2 & 0 \\
\hline 6 & Probable DLB & 65 & M & 3 & 4 \\
\hline 7 & Probable DLB & 70 & M & 5 & 1 \\
\hline 8 & Probable DLB & 61 & M & 4 & 0 \\
\hline 9 & Probable DLB & 81 & $\mathrm{~F}$ & 5 & 1 \\
\hline 10 & Possible DLB & 76 & $\mathrm{~F}$ & 4 & 0 \\
\hline 11 & Probable DLB & 81 & $M$ & 9 & 0 \\
\hline
\end{tabular}

${ }^{*}$ Cognitive fluctuations were assessed with examination described by Walker et al. (19), and apathy was assessed with NPI (20).

$\mathrm{NA}=$ not available.

DLB patients were age-matched with healthy subjects (Table 2), and the MMSE, Grober-Buschke test, Set test, Benton test, visual discrimination test, and Wechsler test scores were significantly lower in DLB patients than in healthy subjects $(P=0.00009,0.0003$, $0.00009,0.00006,0.0053$, and 0.00009 , respectively).

\section{Cholinergic Pathway Integrity Assessment}

The $\mathrm{BP}_{\mathrm{ND}}$ results obtained for the $\mathrm{Ch} 1, \mathrm{Ch} 4$, and $\mathrm{Ch} 5$ pathways are shown in Table 3. In the Ch1 pathway, no significant differences in $\mathrm{BP}_{\mathrm{ND}}$ values were observed between DLB patients and healthy subjects. On the contrary, in the $\mathrm{Ch} 4$ pathway, $\mathrm{BP}_{\mathrm{ND}}$ values for DLB patients were significantly lower in the ACC and in the superior and inferior parietal cortices $(P=0.0006,0.0015$, and 0.0023 , respectively) than those for healthy subjects. $\mathrm{BP}_{\mathrm{ND}}$ values for DLB patients were significantly lower in the $\mathrm{Ch} 5$ terminal region of the thalamus $(P=0.0003)$ and in the striatum $(P=0.0042)$ than those for healthy subjects.

For ROIs showing alterations in cholinergic pathways in DLB, a graphic representation of $\mathrm{BP}_{\mathrm{ND}}$ values obtained for patients with possible DLB $(n=2)$ and patients with probable
DLB $(n=9)$ is shown in Figure 1; the data indicated no observable difference between the 2 groups. For a healthy subject and a representative DLB patient, Figure 2 shows parametric images of simplified $\mathrm{BP}_{\mathrm{ND}}$, a parameter reflecting the ratio of specific binding to nonspecific binding (26), calculated for the striatum and the thalamus from SPECT images obtained $8 \mathrm{~h}$ after injection.

\section{Comparison of Healthy Subjects and DLB Subgroups}

A statistical comparison of $\mathrm{BP}_{\mathrm{ND}}$ values calculated for the ACC and the inferior parietal cortex in the control group, patients with apathy, and patients without apathy is shown in Figure 3. A group effect was found only for the $\mathrm{ACC} ; \mathrm{BP}_{\mathrm{ND}}$ values were significantly lower in patients with apathy than in healthy subjects $(P=0.004)$ and were unchanged in patients without apathy.

\section{DISCUSSION}

The main result of the present study is that, along with an impairment of the striatal cholinergic interneurons, the $\mathrm{Ch} 4$

TABLE 2

Statistical Comparison of Age and Neuropsychological Performance

\begin{tabular}{|c|c|c|c|}
\hline \multirow[b]{2}{*}{ Parameter } & \multicolumn{2}{|c|}{ Median (interquartile range) for: } & \multirow[b]{2}{*}{$P$} \\
\hline & Healthy subjects & DLB patients & \\
\hline Age & $72(6.25)$ & $76(10.50)$ & 0.3376 \\
\hline MMSE score & $29.50(1.25)$ & $21(6.50)$ & $0.00009^{*}$ \\
\hline Grober-Buschke test score & $47.5(2.25)$ & $32(9)$ & $0.0003^{\star}$ \\
\hline Set test score & $76.50(14.50)$ & $33(14.50)$ & $0.00009^{*}$ \\
\hline Benton test score & $14(1.75)$ & $9(5)$ & $0.00006^{\star}$ \\
\hline Visual discrimination test score & $10(0)$ & $9(5.5)$ & $0.0053^{\star}$ \\
\hline Wechsler test code & $43.50(18.50)$ & $10.50(13.25)$ & $0.00009^{*}$ \\
\hline
\end{tabular}


TABLE 3

$\mathrm{BP}_{\mathrm{ND}}$ Values in Each ROI

\begin{tabular}{|c|c|c|c|}
\hline \multirow[b]{2}{*}{$\mathrm{ROI}$} & \multicolumn{2}{|c|}{ Median (interquartile range) for: } & \multirow[b]{2}{*}{$P$} \\
\hline & Healthy subjects $(n=12)$ & DLB patients $(n=11)$ & \\
\hline Ch1: hippocampus & $0.15(0.04)$ & $0.10(0.08)$ & 0.2954 \\
\hline \multicolumn{4}{|l|}{ Ch4 } \\
\hline ACC (BA 24, 32) & $0.17(0.09)$ & $0.07(0.10)$ & $0.0006^{*}$ \\
\hline Posterior cingulate cortex (BA 23) & $0.15(0.07)$ & $0.06(0.14)$ & 0.0074 \\
\hline Prefrontal cortex (BA 11, 12, 9, 44, 45, 46, 47) & $0.19(0.08)$ & $0.17(0.13)$ & 0.8777 \\
\hline Motor and premotor cortices (BA 4, 6, 8) & $0.14(0.07)$ & $0.10(0.11)$ & 0.0110 \\
\hline Occipital cortex (BA 18) & $0.05(0.09)$ & $0.02(0.06)$ & 0.1962 \\
\hline Superior parietal cortex (BA 1, 2, 3, 5, 7) & $0.09(0.04)$ & $0.02(0.05)$ & $0.0015^{\star}$ \\
\hline Inferior parietal cortex (BA 39, 40, 43) & $0.17(0.06)$ & $0.07(0.12)$ & $0.0023^{*}$ \\
\hline Temporal cortex (BA 20, 21, 22, 41, 42) & $0.08(0.07)$ & $0.05(0.06)$ & 0.0150 \\
\hline Ch5: thalamus & $0.45(0.09)$ & $0.14(0.13)$ & $0.0003^{*}$ \\
\hline Striatum & $2.59(0.80)$ & $1.43(0.85)$ & $0.0042^{*}$ \\
\hline
\end{tabular}

${ }^{*} \mathrm{BP}_{\mathrm{ND}}$ values for DLB patients were significantly lower than those for healthy subjects (Mann-Whitney $U$ test; $P<0.0045$ ).

(innominatocortical) and Ch5 (pontothalamic) pathways are altered in DLB, but the Ch1 (septohippocampal) pathway is relatively preserved. Compared with control subjects, patients with apathy exhibited significant cholinergic alterations in the ACC.

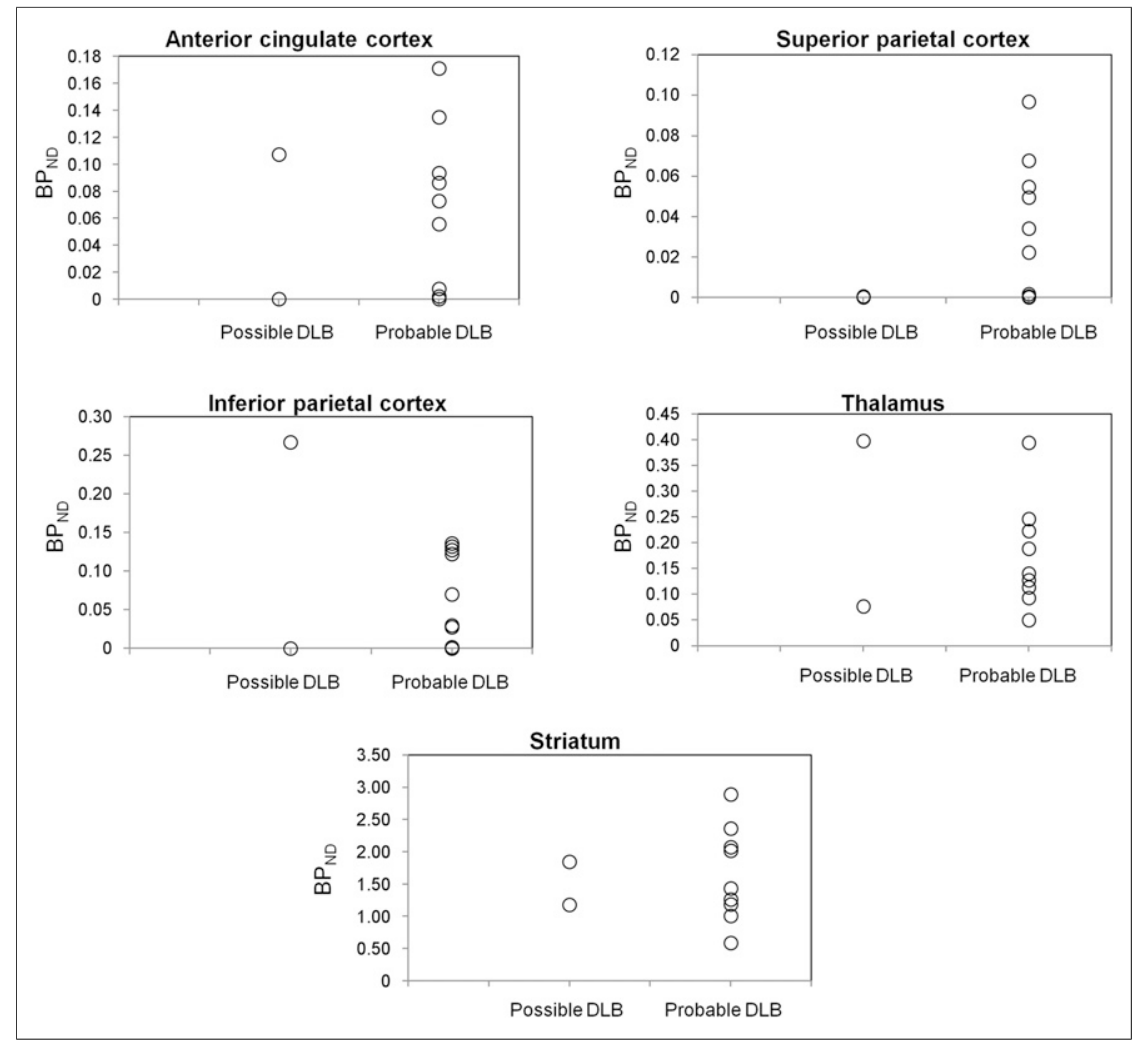

FIGURE 1. Graphic representation of $\mathrm{BP}_{\mathrm{ND}}$ values in ROls showing alterations in cholinergic pathways for patients with possible DLB $(n=2)$ and patients with probable DLB $(n=9)$.
At the subcortical level, our findings of an impaired Ch5 pathway in the thalamus and altered cholinergic interneurons in the striatum are in accordance with postmortem studies showing a reduction in choline acetyltransferase activity in the thalamus (1) and the striatum (27). The present study confirms and complements previous in vivo PET studies as, to date, thalamic cholinergic denervation has been found in only a single small sample of 6 DLB patients (9). In addition, the critical role of the thalamus in DLB was recently highlighted in a multimodal MRI study, and this finding suggested a close relationship between thalamic cholinergic imbalance and fluctuating cognition in DLB (28). At the subcortical level, our findings could provide a differential diagnosis approach with other dementias, such as $\mathrm{AD}$ and parkinsonian dementia, which share clinical features with DLB. Thalamic cholinergic denervation was found in parkinsonian dementia and DLB but not in AD (9). Thus, the evaluation of thalamic cholinergic innervation itself does not allow the differentiation of DLB from other degenerative dementias; in this regard, an assessment of striatal cholinergic integrity may provide valuable information. Indeed, until now, striatal cholinergic innervation remained unexplored in vivo in DLB.

Our ${ }^{123}$ I-iodobenzovesamicol study complements previous in vivo work by Kuhl et al. (13), who used ${ }^{123}$ I-iodobenzovesamicol in $\mathrm{AD}$ and Parkinson disease patients and who found an unchanged striatal cholinergic status in both $\mathrm{AD}$ patients and Parkinson disease patients with and without dementia. As dopamine and acetylcholine strongly 


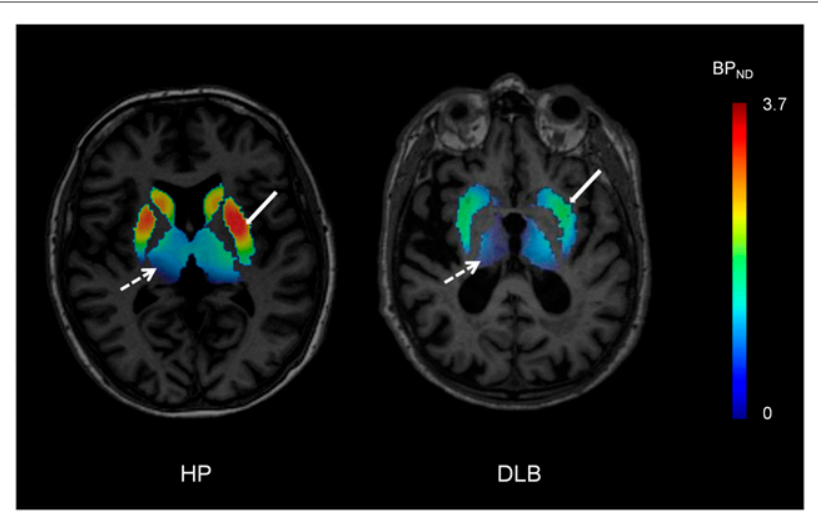

FIGURE 2. Representative simplified $\mathrm{BP}_{\mathrm{ND}}$ parametric images acquired $8 \mathrm{~h}$ after injection (transverse view) from healthy participant $(\mathrm{HP})$ and patient with DLB. Simplified $\mathrm{BP}_{\mathrm{ND}}$ values (ratio of specific binding to nonspecific binding) in striatum (solid arrows) and thalamus (broken arrows) were calculated from SPECT images as [ROI activity concentration (i.e., total binding) - reference region activity concentration (i.e., nonspecific binding)/reference region activity concentration] and were overlaid on MR image for each subject. Ratio of specific binding to nonspecific binding was visually lower in striatum and thalamus for patient with DLB than for healthy participant.

interact in the striatum, differential alterations of the balance between acetylcholine and dopamine in the striatum may explain the differences observed between the Parkinson disease patients in the study of Kuhl et al. (13) and the DLB patients in the present study. For each of these neurologic disorders, a dual molecular imaging approach exploring both neurotransmitters in the striatum would allow verification of this hypothesis. Altogether, the results of the present study and the data of Kuhl et al. (13) show that, in vivo, striatal cholinergic alterations are found in DLB but not in AD and parkinsonian dementia. These findings provide evidence that in vivo ${ }^{123} \mathrm{I}$-iodobenzovesamicol SPECT imaging may be a valuable tool for differentiating DLB patients from $\mathrm{AD}$ patients and Parkinson disease patients with dementia.

Our findings seem to clarify conflicting results from previous PET studies that assessed the integrity of cortical cholinergic innervation in DLB. One recent in vivo study showed variable alterations in cortical AChE activity (29), whereas 3 others showed a widespread and profound reduction in cortical AChE activity (6-8), especially in the posterior regions of the parietooccipital cortices. Because they demonstrate the existence of cholinergic damage in the superior and inferior parietal cortices, our results are in good agreement with these findings, except in the occipital regions, probably because of

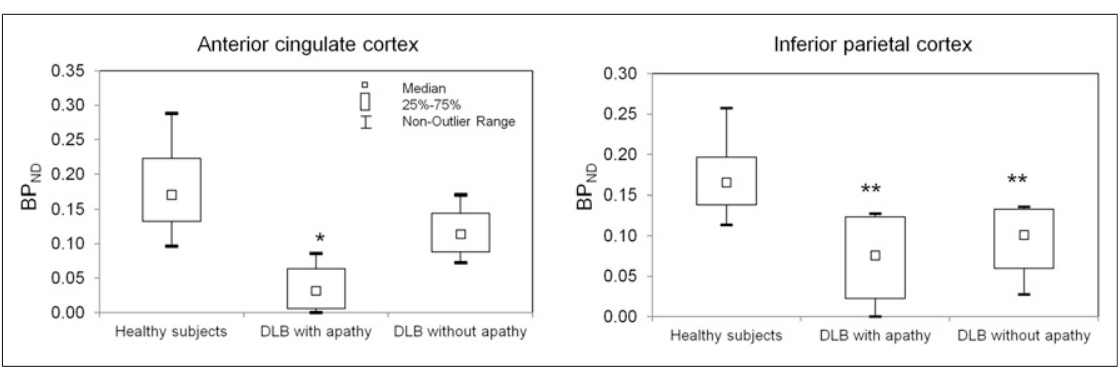

FIGURE 3. $\mathrm{BP}_{\mathrm{ND}}$ values calculated for $\mathrm{ACC}$ and inferior parietal cortex in healthy subjects, patients with apathy, and patients without apathy. ${ }^{*} P=0.004$ for patients vs. healthy subjects. ${ }^{\star \star} P=0.0008$ for patients vs. healthy subjects. differences in the tracers used. From a physiologic point of view, VAChT density is very low in the occipital cortex $(24,26)$, making a comparison of this structure in patients and control subjects with ${ }^{123}$ I-iodobenzovesamicol difficult. Furthermore, noncholinergic (i.e., choline acetyltransferase- or VAChT-negative) but AChE-rich neurons (i.e., cholinoceptive neurons) are found in almost all parts of the brain (30), providing another explanation for the more widespread cortical alterations found in previous PET studies than in the present study. The results of the present study are also congruent with data provided by SPECT perfusion imaging, which revealed a perfusion deficit in the inferior parietal cortex of DLB patients; this finding may underlie the visuospatial perception deficit in DLB (31). Additionally, spatial congruence of deficits in AChE activity and ${ }^{18} \mathrm{~F}-\mathrm{FDG}$ hypometabolism occurred in the parietooccipital regions of DLB patients (6).

Our results extend earlier data by revealing that cholinergic neuronal loss occurs in the ACC of DLB patients. In the present study, compared with control subjects, patients with apathy exhibited a significant cholinergic alteration in this cortical structure, and a trend toward lower $\mathrm{BP}_{\mathrm{ND}}$ values was observed in patients with apathy but not in patients without apathy. The refusal of 3 individuals to take the examination described by Walker et al. (19) and the NPI examination, apart from the fact that it decreased the number of data, could have been a selection bias in the sense that refusal could reflect a particular personality profile that might be related to the degree of pathologic damage.

Although the findings of the present study must be confirmed with a larger sample of patients, they may provide evidence that alterations in cholinergic transmission in the ACC could be closely associated with the development of apathy. From a functional point of view, cholinergic neurons of the nucleus basalis of Meynert provide the major source of cortical cholinergic innervation through the $\mathrm{Ch} 4$ pathway and exhibit strong positive connectivity with a network of brain regions that orchestrate goal-directed behavior, particularly in the ACC (32). The possible role of acetylcholine in apathy has also been documented in animal and pharmacologic studies, as the positive effect of cholinergic drugs on apathy has been reported in animal models of apathy (33). In DLB, apathy, hallucinations, and sleep disorders are some of the most frequently cited treatment-responsive symptoms after treatment with cholinesterase inhibitor drugs $(1,34)$. In addition, the ACC has extensive projections to the amygdala, ventral striatum, and nucleus accumbens, and this circuit is important for goal-directed and motivated behaviors $(32,35)$. Other neuroimaging studies, including PET measures of ${ }^{18} \mathrm{~F}-\mathrm{FDG}$ metabolism and structural brain changes on MRI, have also supported the role of ACC in apathy (32,36,37).

Some limitations of the present study merit consideration. First, the small number of investigated patients did not allow us to significantly discriminate patients with apathy from those without apathy on the basis of ${ }^{123}$ I-iodobenzovesamicol in the ACC. Second, in the ROIs showing alterations of the cholinergic pathways in DLB, no difference in $\mathrm{BP}_{\mathrm{ND}}$ values was observed between patients with possible DLB and patients with probable DLB; this finding suggested that the cholinergic system is similarly affected in all patients, irrespective of the course of the disease. However, in other brain areas, where no significant cholinergic alteration was found, we cannot exclude the possibility that cholinergic deficits were masked by the presence of two patients with possible DLB. Finally, although a fully automated and quantitative method was used, the 
low resolution of SPECT imaging can represent a limitation to studying thin gray matter areas, such as the cortical structures. In this regard, ${ }^{18} \mathrm{~F}$-fluoroethoxybenzovesamicol (38) could be a useful PET radioligand for exploring cholinergic integrity in larger groups of DLB patients.

\section{CONCLUSION}

The results of the present study support, for DLB in vivo, a general cholinergic neuronal loss, reaching both cortical and subcortical levels, with the exception of the Ch1 pathway. Our results confirmed the findings of previous postmortem studies and showed-to our knowledge, for the first time, in vivo- the alterations of cholinergic interneurons in the striatum in DLB. Given our results and those of Kuhl et al. (13), ${ }^{123}$ I-iodobenzovesamicol appears to be a valuable tool for differentiating DLB from both $\mathrm{AD}$ and parkinsonian dementia. As the reciprocal balance of dopamine and acetylcholine in the striatum is well known and given the parallel alteration of these neurotransmitters in DLB, it would be interesting to explore more thoroughly in vivo in DLB patients both cholinergic and dopaminergic neurotransmission to provide a better understanding of the relationship between these 2 neurotransmitters in DLB.

\section{DISCLOSURE}

No potential conflict of interest relevant to this article was reported.

\section{ACKNOWLEDGMENTS}

We thank Caroline Buisson and Thierry Blandin for technical assistance.

\section{REFERENCES}

1. O'Brien J, Ames D, McKeith I, Chiu E. Dementia with Lewy Bodies and Parkinson's Disease Dementia. Oxford, United Kingdom: Taylor and Francis; 2006:33-48.

2. McKeith IG, Dickson DW, Lowe J, et al. Diagnosis and management of dementia with Lewy bodies: third report of the DLB Consortium. Neurology. 2005;65:1863-1872.

3. McKeith I, O'Brien J, Walker Z, et al. Sensitivity and specificity of dopamine transporter imaging with ${ }^{123}$ I-FP-CIT SPECT in dementia with Lewy bodies: a phase III, multicentre study. Lancet Neurol. 2007;6:305-313.

4. Perry EK, Haroutunian V, Davis KL, et al. Neocortical cholinergic activities differentiate Lewy body dementia from classical Alzheimer's disease. Neuroreport. 1994;5:747-749.

5. Tiraboschi P, Hansen LA, Alford M, et al. Early and widespread cholinergic losses differentiate dementia with Lewy bodies from Alzheimer disease. Arch Gen Psychiatry. 2002;59:946-951.

6. Klein JC, Eggers C, Kalbe E, et al. Neurotransmitter changes in dementia with Lewy bodies and Parkinson disease dementia in vivo. Neurology. 2010;74:885-892.

7. Shimada H, Hirano S, Shinotoh H, et al. Mapping of brain acetylcholinesterase alterations in Lewy body disease by PET. Neurology. 2009;73:273-278.

8. Herholz K, Bauer B, Wienhard K, et al. In-vivo measurements of regional acetylcholine esterase activity in degenerative dementia: comparison with blood flow and glucose metabolism. J Neural Transm. 2000;107:1457-1468.

9. Kotagal V, Muller ML, Kaufer DI, Koeppe RA, Bohnen NI. Thalamic cholinergic innervation is spared in Alzheimer disease compared to parkinsonian disorders. Neurosci Lett. 2012;514:169-172.

10. Mazère J, Meissner WG, Mayo W, et al. Progressive supranuclear palsy: in vivo SPECT imaging of presynaptic vesicular acetylcholine transporter with [ $\left.{ }^{123} \mathrm{I}\right]-$ iodobenzovesamicol. Radiology. 2012;265:537-543.

11. Mazere J, Meissner WG, Sibon I, et al. [ ${ }^{123}$ I]-IBVM SPECT imaging of cholinergic systems in multiple system atrophy: a specific alteration of the pontothalamic cholinergic pathways (Ch5-Ch6). Neuroimage Clin. 2013;3:212-217.

12. Mazère $\mathrm{J}$, Prunier $\mathrm{C}$, Barret $\mathrm{O}$, et al. In vivo SPECT imaging of vesicular acetylcholine transporter using $\left[{ }^{123} \mathrm{I}\right]-\mathrm{IBVM}$ in early Alzheimer's disease. Neuroimage. 2008;40:280-288.
13. Kuhl DE, Minoshima S, Fessler JA, et al. In vivo mapping of cholinergic terminals in normal aging, Alzheimer's disease, and Parkinson's disease. Ann Neurol. 1996;40:399-410.

14. Folstein MF, Folstein SE, McHugh PR. "Mini-mental state": a practical method for grading the cognitive state of patients for the clinician. J Psychiatr Res. 1975;12:189-198.

15. Grober E, Buschke H, Crystal H, Bang S, Dresner R. Screening for dementia by memory testing. Neurology. 1988;38:900-903.

16. Benton A. The Revised Vision Retention Test. 3rd ed. New York, NY: Psychological Corp.; 1963.

17. Isaacs B, Kennie AT. The Set test as an aid to the detection of dementia in old people. Br J Psychiatry. 1973;123:467-470.

18. Wechsler D. Wechsler Adult Intelligence Scale Manual. New York, NY: Psychological Corp.; 1981.

19. Walker MP, Ayre GA, Cummings JL, et al. The Clinical Assessment of Fluctuation and the One Day Fluctuation Assessment Scale: two methods to assess fluctuating confusion in dementia. Br J Psychiatry. 2000;177:252-256.

20. Cummings JL, Mega M, Gray K, Rosenberg-Thompson S, Carusi DA, Gornbein J. The Neuropsychiatric Inventory: comprehensive assessment of psychopathology in dementia. Neurology. 1994;44:2308-2314.

21. Jung YW, Frey KA, Mulholland GK, et al. Vesamicol receptor mapping of brain cholinergic neurons with radioiodine-labeled positional isomers of benzovesamicol. J Med Chem. 1996;39:3331-3342.

22. Müller-Gärtner HW, Links JM, Prince JL, et al. Measurement of radiotracer concentration in brain gray matter using positron emission tomography: MRI-based correction for partial volume effects. J Cereb Blood Flow Metab. 1992;12:571-583.

23. Fischl B, Salat DH, Busa E, et al. Whole brain segmentation: automated labeling of neuroanatomical structures in the human brain. Neuron. 2002;33:341-355.

24. Barret O, Mazere J, Seibyl J, Allard M. Comparison of noninvasive quantification methods of in vivo vesicular acetylcholine transporter using $\left[{ }^{123} \mathrm{I}\right]-\mathrm{IBVM}$ SPECT imaging. J Cereb Blood Flow Metab. 2008;28:1624-1634.

25. Ichise M, Liow JS, Lu JQ, et al. Linearized reference tissue parametric imaging methods: application to $\left[{ }^{11} \mathrm{C}\right] \mathrm{DASB}$ positron emission tomography studies of the serotonin transporter in human brain. J Cereb Blood Flow Metab. 2003;23:1096-1112.

26. Mazère J, Mayo W, Pariscoat G, et al. Simplified quantification method for in vivo SPECT imaging of the vesicular acetylcholine transporter with ${ }^{123} \mathrm{I}$ iodobenzovesamicol. J Nucl Med. 2015;56:862-868.

27. Langlais PJ, Thal L, Hansen L, Galasko D, Alford M, Masliah E. Neurotransmitters in basal ganglia and cortex of Alzheimer's disease with and without Lewy bodies. Neurology. 1993;43:1927-1934.

28. Delli Pizzi S, Franciotti R, Taylor JP, et al. Thalamic involvement in fluctuating cognition in dementia with Lewy bodies: magnetic resonance evidences. Cereb Cortex. 2015;25:3682-3689.

29. Marcone A, Garibotto V, Moresco RM, et al. [ $\left.{ }^{11} \mathrm{C}\right]-\mathrm{MP} 4 \mathrm{~A}$ PET cholinergic measurements in amnestic mild cognitive impairment, probable Alzheimer's disease, and dementia with Lewy bodies: a Bayesian method and voxel-based analysis. J Alzheimers Dis. 2012;31:387-399.

30. Giacobini E. Cholinesterases and Cholinesterase Inhibitors. London, United Kingdom: Martin Dunitz Ltd.; 2000.

31. Firbank MJ, Colloby SJ, Burn DJ, McKeith IG, O'Brien JT. Regional cerebral blood flow in Parkinson's disease with and without dementia. Neuroimage. 2003;20:1309-1319.

32. Pinto T, Lanctot KL, Herrmann N. Revisiting the cholinergic hypothesis of behavioral and psychological symptoms in dementia of the Alzheimer's type. Ageing Res Rev. 2011;10:404-412.

33. Martinowich K, Cardinale KM, Schloesser RJ, Hsu M, Greig NH, Manji HK. Acetylcholinesterase inhibition ameliorates deficits in motivational drive. Behav Brain Funct. 2012;8:15.

34. Cummings J, Lai TJ, Hemrungrojn S, et al. Role of donepezil in the management of neuropsychiatric symptoms in Alzheimer's disease and dementia with Lewy bodies. CNS Neurosci Ther. 2016;22:159-166.

35. Parvizi J, Rangarajan V, Shirer WR, Desai N, Greicius MD. The will to persevere induced by electrical stimulation of the human cingulate gyrus. Neuron. 2013;80: 1359-1367.

36. Apostolova LG, Akopyan GG, Partiali N, et al. Structural correlates of apathy in Alzheimer's disease. Dement Geriatr Cogn Disord. 2007;24:91-97.

37. Marshall GA, Monserratt L, Harwood D, Mandelkern M, Cummings JL, Sultzer DL. Positron emission tomography metabolic correlates of apathy in Alzheimer disease. Arch Neurol. 2007;64:1015-1020.

38. Petrou M, Frey KA, Kilbourn MR, et al. In vivo imaging of human cholinergic nerve terminals with (-)-5- ${ }^{18} \mathrm{~F}$-fluoroethoxybenzovesamicol: biodistribution, dosimetry, and tracer kinetic analyses. J Nucl Med. 2014;55:396-404. 